\title{
The Adoption Problem and Anti-Exceptionalism about Logic
}

\author{
Suki Finn
}

\begin{abstract}
Anti-exceptionalism about logic takes logic to be, as the name suggests, unexceptional. Rather, in naturalist fashion, the anti-exceptionalist takes logic to be continuous with science, and considers logical theories to be adoptable and revisable accordingly. On the other hand, the Adoption Problem aims to show that there is something special about logic that sets it apart from scientific theories, such that it cannot be adopted in the way the anti-exceptionalist proposes. In this paper I assess the damage the Adoption Problem causes for anti-exceptionalism, and show that it is also problematic for exceptionalist positions too. My diagnosis of why the Adoption Problem affects both positions is that the self-governance of basic logical rules of inference prevents them from being adoptable, regardless of whether logic is exceptional or not.
\end{abstract}

Keywords: Anti-exceptionalism; adoption; rule-following; inference; rule-circularity.

\section{Where it all began: Lewis Carroll}

Once upon a time, in 1895, Lewis Carroll published an influential puzzle about the epistemology of logic, in a short paper named 'What the Tortoise Said to Achilles'. In this puzzle, the Tortoise refuses to accept conclusion $Z$ ('The two sides of this Triangle are equal to each other') on the basis of the premises $A$ ('Things that are equal to the same are equal to each other') and $B$ ('The two sides of this Triangle are things that are equal to the same'). ${ }^{1}$ The Tortoise accepts $A$ and $B$ but does not feel forced to accept $Z$. It is as if the Tortoise is driving a wedge between $A$ and $B$ on one side, and $Z$ on the other (although this is not how Carroll phrases it). Achilles tries to get

${ }^{1}$ Carroll (1895) p278

Australasian Journal of Logic (16:7) 2019, Article no. 3 
the Tortoise to accept $Z$, and so asks them to consider and accept another premise, $C$ ('If $A$ and $B$ are true, $Z$ must be true'), in order to fill in the gap and forge a bridge to $Z{ }^{2}$ However, this leads to an infinite regress of added premises, since without also adding $D$ ('If $A$ and $B$ and $C$ are true, $Z$ must be true'), and $E$ ('If $A$ and $B$ and $C$ and $D$ are true, $Z$ must be true'), etc., there is still a gulf in between the premises and $Z$. Achilles will not move the Tortoise to $Z$ by adding these premises as the gulf cannot be breached by inserting them - they simply do not help. One lesson to learn then from this puzzle is that we cannot use the very same inference rules utilized in the argument as premises in the argument.

There are many ways to understand what the Tortoise taught us in Carroll's puzzle, of which I shall discuss two: Quine's and Kripke's interpretations with relation to anti-exceptionalism. Quine famously used the puzzle as an objection against Carnap to show that logical rules cannot be analytically true by convention. ${ }^{3}$ More recently, Kripke uses the puzzle as an objection against Quine to show that logical rules cannot be empirical and unprivileged. ${ }^{4}$ Kripke takes his objection to Quine to be 'exactly the same' as the objection that Quine makes to Carnap, thus entailing that Kripke's objection should apply to Carnap as well. Padró interprets Kripke's use of Carroll's puzzle as what she calls the Adoption Problem. ${ }^{6}$ This problem will be the main focus of my paper.

The Adoption Problem (hereon 'AP') delivers a fatal blow to the antiexceptionalist, for whom the logical laws are empirical and unprivileged. ${ }^{7}$ However, I will show how the AP is indifferent to whether we take the logical rules to be empirical or analytic, demonstrating that the AP does not discriminate among different interpretations of the status or justification of the logical rules. Rather, there is a far more fundamental issue that the AP exposes that cannot be resolved by appeal to how we justify our logic or by the level of privilege we give to it. This fundamental issue is the role that the logical rules play in our practice of inferring, caused by the self-governing nature that the basic rules of logic encounter, and this self-governance will be problematic regardless of whether one is an anti-exceptionalist or not. The

\footnotetext{
${ }^{2}$ Ibid. p279

${ }^{3}$ Quine (1936)

${ }^{4}$ This is currently unpublished but is described in Kripke (1974a) \& (1974b), and Padró (2015).

${ }^{5}$ Kripke (1974a) as quoted in Padró (2015) p113.

${ }^{6}$ This is also currently unpublished, but is described in Padró's (2015).

${ }^{7} \mathrm{My}$ understanding of the anti-exceptionalist position is primarily based on Hjortland (2017).
} 
self-governance of the basic logical rules of inference is what is at the heart of the AP, and it is this observation that comprises my unique diagnosis of the problem.

\section{The rise of anti-exceptionalism: Quine vs Carnap}

Exceptionalism about logic is the position that holds that logic is a priori and analytic. Anti-exceptionalism on the other hand holds that "logic isn't a priori, nor are its truths analytic truths." 8 Importantly, the exceptionalist takes logic to be exceptional in some way such that it is unlike science, whereas the anti-exceptionalist takes logic to be unexceptional such that it is continuous with science. For the purposes of this paper, I take Quine to be the quintessential anti-exceptionalist, ${ }^{9}$ arguing against Carnap who will represent the exceptionalist. ${ }^{10}$ At least until the recent resurgence of attempts to revive Carnapian exceptionalism, it was fair to say at the end of the 20th century that the consensus was that Quine had won the debate, and the prospects of a Carnapian style of exceptionalism about logic were buried as a result. ${ }^{11}$

Carnap, of The Logical Syntax of Language, is said to hold a position where logical truths are necessary and known a priori, grounded on linguistic conventions. ${ }^{12}$ On this interpretation, logic is such that it is true by virtue of meaning, where its statements are considered the product of linguistic conventions. So, on this picture there will be prescriptive stipulated conventions of how we infer, and rules of inference will become valid or justified in virtue of these conventions. Logical truths are therefore established by means of conventional stipulations, and are unlike scientific truths in that they are not about the world. This is sufficiently in line with the exceptionalist view for our sake, and given that this is the interpretation of Carnap that Padró

\footnotetext{
${ }^{8}$ Hjortland (2017) p631

${ }^{9}$ It is taken as being uncontroversial that Quine (1951) is an anti-exceptionalist. As Hjortland notes, Quine is anti-exceptionalism's "most famous proponent". Hjortland (2017) p631

${ }^{10}$ Carnap (1937) endorses a sufficient amount of the tenants of exceptionalism to qualify as an exceptionalist, particularly with regard to the aspects that Quine (1951) disagrees with.

${ }^{11}$ Ebbs (2011) gives multiple examples of where this standard story has been assumed in the literature. Yablo (1998) p232 also states "That Carnap is widely seen to have lost the ensuing debate is a fact from which the quizzical camp has never quite recovered".

${ }^{12}$ See Carnap (1937) p1 where he challenges the distinction between rules of logic and rules of syntax and says the logical characteristics of sentences are grounded in their syntactic character.
}

Australasian Journal of Logic (16:7) 2019, Article no. 3 
works with, ${ }^{13}$ I hereon refer to the Carnapian as the exceptionalist position that is of relevance to Padró's AP.

In 'Truth by Convention', Quine puts forward his famous objection against Carnap, that the laws of logic cannot be true by convention (or in other words, they cannot be purely analytic). Quine argues that the laws of logic cannot be justified in this way, as they would require those same laws to derive the laws from the conventions which in turn require justification, resulting in a regress similar to that demonstrated by Carroll in 'What the Tortoise said to Achilles'. Quine's attack starts by acknowledging that there are infinitely many logical truths, and so the logical truths cannot be stipulated one by one, but rather must be given by general conventions. But to move from a general convention to a particular case requires further inference. If this inference rule is also a result of stipulation then we embark on a regress, which Quine thinks is analogous to Carroll's regress. This initial attack on the notion of truth by convention is developed further in Quine (1951) in the form of his full-blown attack on the analytic, and from thereon began the rise of Quinean anti-exceptionalism that takes logic to be empirical and on a par with science.

\section{The fall of anti-exceptionalism: Kripke vs Quine}

Despite Quine seemingly devastating the Carnapian conventionalist position using Carroll's puzzle, Quine himself was not immune to the puzzle's potency. As Kripke shows, Carroll's puzzle can similarly be utilized against Quine's position too. And so what led to the rise of anti-exceptionalism also leads to its fall. According to Kripke then, and apparently unbeknownst to Quine, Quine's own argument against Carnap works just as well against Quine himself:

I cannot for the life of me, see how [Quine] criticizes [Carnap's] view and then presents an alternative which seems to me to be subject to exactly the same difficulty. ${ }^{14}$

\footnotetext{
${ }^{13}$ See Padró (2015) p87: "For Carnap, the a prioricity and necessity of logic, mathematics, and conceptual truths was grounded on linguistic conventions." (Emphasis in original).

${ }^{14}$ Kripke (1974a) as quoted in Padró (2015) p113. Padró (2015) argues that there are significant differences (despite the similarities) between (i) Carroll's puzzle, (ii) Quine's objection to Carnap, and (iii) Kripke's objection to Quine, such that they are not 'exactly the same difficulty'. Padró articulates Kripke's objection and names it the 'Adoption Problem', which I argue under close scrutiny reveals the common root of (i)-(iii) to be the self-governing nature of the basic logical rules, which is indifferent to exceptionalism/antiexceptionalism.
}

Australasian Journal of Logic (16:7) 2019, Article no. 3 
The alternative view to Carnap's exceptionalism that Quine puts forward is the anti-exceptionalist position from his 'Two Dogmas of Empiricism'. ${ }^{15}$ And so despite Quine and Carnap holding different views about the nature of logic, they are both susceptible to the same problem deriving from Carroll's puzzle.

In 'Two Dogmas of Empiricism', Quine famously blurs the analytic/synthetic distinction. This removes any sharp division between logical principles and empirical hypotheses, as neither will have a privileged status in one's web of belief. Rather there will be a gradation across the web, with logic residing closer to the center. Logical principles, like empirical hypotheses, can be revisable in the face of some recalcitrant experience, and we importantly have a choice regarding which it is that we revise. ${ }^{16}$ Logic thus becomes justified in a holistic way by coping with experience, just like everything else is justified. The laws of logic will have no special status over and above other elements of the system, and so those laws are to be considered empirical, as opposed to analytic as Carnap would have it. Given that on Quine's view the logical laws are not grounded in conventions as they are on Carnap's, one might have assumed that Quine could escape the problems that Carnap encountered. However, for the same reasons that Quine argued that Carnap could not deduce anything from the logical laws if they are conventional, Kripke argues that Quine could not deduce anything from them if they are empirical:

It seems to me, as I said last time, obviously to go just as strongly against Quine's own statements that logical laws are just hypotheses within the system which we accept just like any other laws, because then, too, how is one going to deduce anything from them $?^{17}$

In answer to that question, Kripke claims that one cannot deduce anything from the logical laws without having those logical laws in advance to appeal to:

\footnotetext{
${ }^{15}$ Notice that it is the 'Quine' from 1936 that attacks Carnap, yet the 'Quine' from 1951 that gets attacked by Kripke on the same basis. The positions held by Quine in 1936 and 1951 may be different and even incompatible with each other, but that is a story for a different paper.

${ }^{16}$ On this, Kripke (1974b) argues against Putnam (1969) who applies Quine's method with all seriousness and proposes to revise logic for quantum mechanics. Kripke thus attacks Putnam regarding the empirical nature of logic, and attacks Quine regarding logic's unprivileged status.

${ }^{17}$ Kripke (1974a) as quoted in Padró (2015) p113.
} 
Logic, even if one tried to throw intuitions to the wind, cannot be like geometry, because one cannot adopt the logical laws as hypotheses and draw their consequences. You need logic in order to draw these consequences. There can be no neutral ground in which to discuss the drawing of consequences independently of logic itself. ${ }^{18}$

In Quine's naturalistic setting, Kripke argues the logical laws have no scientific fertility due to having 'never led to a single prediction'. ${ }^{19}$ Without granting the logical laws a special status, they will be understood as hypotheses that we adopt in order to determine their empirical impact. But their empirical impact will be zero as they will not be able to imply anything, due to requiring logical laws themselves in order to deduce anything from them (as shown by Quine's use of Carroll's puzzle against Carnap). Kripke's objection is thus directed at the unprivileged and empirical status of logical laws, and so we can treat Kripke's usage of Carroll's puzzle as an attack against the anti-exceptionalist.

What we have seen so far is that both Quine and Kripke claim to be putting forward an objection related to Carroll's puzzle, yet they use it to attack opposite targets. Quine's usage of the puzzle attacks Carnap's exceptionalist view of logic as being conventional, and Kripke's usage of the puzzle attacks Quine's anti-exceptionalist view of logic as being empirical. If the objections from Quine and Kripke are 'exactly the same' (as Kripke states $)^{20}$, then this shows that the problem derived from Carroll's puzzle does not discriminate amongst its targets with regard to the status of logical laws. Yet despite any differences that one may note between Quine and Kripke's attacks, ${ }^{21}$ both have in common the problem of being able to deduce anything from the laws of logic without using the laws of logic themselves. I argue that this unifies the objections and is due to the self-governance of such rules. Therefore, despite the AP being explicitly put forward by Kripke as an objection against Quinean anti-exceptionalism, the AP can strike just as hard against exceptionalism too.

\footnotetext{
${ }^{18}$ Kripke (1974b) as quoted in Padró (2015) p140. Kripke is here remarking on Putnam (1969).

${ }^{19}$ Kripke (1974a) as quoted in Padró (2015) p112.

${ }^{20}$ Yet stating the objections are the same when they have different targets seems inconsistent.

${ }^{21}$ Padró (2015 chap.4) describes the differences, and notes the similarity between Carnap's and Quine's views leaving them open to such objections is that both aim to justify the logical laws.
}

Australasian Journal of Logic (16:7) 2019, Article no. 3 


\section{The Adoption Problem}

The Adoption Problem (AP) was coined by Padró, and is Padró's articulation of Kripke's objection to Quine. ${ }^{22}$ Given that the objection was based on Kripke's interpretation of Carroll's 'What the Tortoise said to Achilles', the AP captures 'what the Tortoise said to Kripke'. ${ }^{23}$ Kripke describes the AP as such:

There are certain rules which you just couldn't adopt: you couldn't tell them to yourself, because if you told them to yourself without already using them, they would be useless; so they either don't help you or they were superfluous anyway. ${ }^{24}$

And in Padró's words, the AP can be understood as the following dilemma:

Certain basic logical principles cannot be adopted, because, if a subject already infers in accordance with them, no adoption is needed, and if the subject does not infer in accordance with them, no adoption is possible. ${ }^{25}$

So why is it that certain logical rules are useless if we do not already infer in accordance with them? Surely, if we do not already use them, then adopting such rules would be useful, so that we could come to use them. But according to the AP, this is not possible. I will show that the reason why is due to such rules being self-governing (being of the very structure that the rule itself aims to govern, thus requiring an application of itself in order to be used). It is this feature of self-governance that prevents the logical rules from being adoptable, and it is also this that underlies Carroll's puzzle and Quine's attack on Carnap. A better understanding of the AP therefore will help us to identify the unifying aspects of Kripke and Quine's objections, in order to show that the underlying problem is not particular to anti-exceptionalist or exceptionalist views of logic.

The AP is best understood using the story of Harry and the raven. ${ }^{26}$ Harry is special — he has never heard of, nor inferred in accordance with,

\footnotetext{
${ }^{22}$ Padró (2015)

${ }^{23}$ This is Padró's phrase, and is the title of her 2015 thesis.

${ }^{24}$ Kripke (1974a) as quoted in Padró (2015) p112.

${ }^{25}$ Padró (2015) p42. Padró speaks of logical principles in this quote, but in the previous quote Kripke speaks of logical rules. In this paper, particularly in section 4, I focus on logical rules.

${ }^{26}$ See Padró (2015) and Kripke (1974b).
} 
the logical rule of Universal Instantiation (hereon 'UI'). A problem arises when we invite Harry to pick up the practice of inferring according to UI, by means of accepting UI itself. We may think that Harry would be better off inferring according to UI rather than not, but we cannot help Harry attain this practice by simply giving him the UI rule. Kripke outlines this in the following example:

I say to him, 'Consider the hypothesis that from each universal statement, each instance follows'. Now, previously to being told this, he believed it when I said that all ravens are black because I told him that too. But he was unable to infer that this raven, which is locked in a dark room, and he can't see it, is therefore black. And in fact, he doesn't see that that follows, or he doesn't see that that is actually true. So I say to him, 'Oh, you don't see that? Well, let me tell you, from every universal statement each instance follows.' He will say, 'Okay, yes. I believe you.' Now I say to him, "All ravens are black" is a universal statement, and 'This raven is black' is an instance. Yes?' 'Yes,' he agrees. So I say, 'Since all universal statements imply their instances, this particular universal statement, that all ravens are black, implies this particular instance.' He responds: 'Well, Hmm, I'm not entirely sure. I don't really think that I've got to accept that. ${ }^{27}$

What is going on here is that Harry would already need to be able to make UI inferences in order to grasp that the UI rule applies to this case of ravens. But Harry cannot apply it here, since that would presuppose an inferential move from a general rule to a particular case, which is an UI inference. This is due to UI being of universal form, in that it is a general rule that applies to all cases of universals: for all universals, you may draw the instances. As such, UI is self-governing, by being of the form that it governs. Since Harry does not know what to do with universals, giving him a rule that governs universals that is itself in the form of a universal is not going to help him. He needs to use UI in order to apply UI to a case, and so needs to apply it twice. We do not help Harry by giving him the UI rule as if he did not universally instantiate in the first place then he certainly will not now be able to do it twice. He would first need to derive that the case about ravens is one which the rule applies to (which utilizes the UI rule), and then derive the colour

\footnotetext{
${ }^{27}$ Kripke (1974b) as quoted in Padró (2015) p35. (Emphasis in original.) Note that 'Harry' is the name used in Padró's example, and in Kripke's example a different subject is used.
} 
of the particular raven (which also utilizes the UI rule). So if Harry did not already infer according to UI, just telling him that UI is true would not be of any use to him. ${ }^{28}$

This is clearly reminiscent of Carroll's puzzle and Quine's objection against Carnap. We once again see that nothing can be derived from the logical rules, such that they cannot be put to practical use, due to the self-governing nature of the rule in question. Carroll's Tortoise battles with Modus Ponens (hereon 'MP'), which is also self-governing. This is because MP tells us how to derive a conclusion from a conditional, but is itself a conditional, as it tells us what to do if faced with a conditional and its antecedent. ${ }^{29}$ The Tortoise refuses to accept $Z$ on the basis of $A$ and $B$, where if $A$ and $B$ are true then $Z$ is true. MP does not help the Tortoise, as MP is a conditional, thus is of the form that she doesn't infer in accordance with. The Tortoise does not respond appropriately to conditionals, so giving her a rule that says how to respond to conditionals that is itself a conditional is not going to move her to infer accordingly.

I argue that MP and UI have something in common which prevents them from being adoptable. ${ }^{30}$ Namely these rules govern such basic and fundamental patterns of inference that they underwrite the application of any logical rule, including themselves. As such, they both govern their own application. I will show this by looking at the general structure of logical rules of inference to demonstrate how such structure presupposes MP and UI. Logical rules of inference are, very generally speaking, universals and conditionals in their structure. To be of a universal structure is to apply in all cases of a certain kind. To be conditional in structure is to say what to do if in a case of a certain kind.

Logical rules of inference take us from premises to a conclusion via a conditional, and are universal so that they apply in all cases when the antecedent of that conditional is satisfied. The antecedent of the conditional will name a situation when the rule is applicable, and the consequent of the conditional will name what one should do when faced with an instance of that situation. It is therefore written in to the very nature of what it is to be a logical rule of inference that it is universal and conditional in its structure. So given that we have said that all logical rules of inference are universal and conditional in their structure, then those rules that govern or describe how to deal with universal or conditional structures (namely UI

\footnotetext{
${ }^{28}$ This is described in Kripke (1974b) and in Padró (2015).

${ }^{29}$ I have described the self-governing nature of MP and UI in my (2019).

${ }^{30}$ Similar issues would arise for dropping UI and MP, as described in Padró (2015) p61-62.
}

Australasian Journal of Logic (16:7) 2019, Article no. 3 
and MP respectively) will face problems by being of the structure that they themselves govern. Thus UI and MP will unavoidably govern themselves, due to always being logical rules with the structure that they themselves govern. Logical rules seem to presuppose that we can make sense of instructions of the form 'if...then', and recognise instances as instances of general forms. Therefore some grasp of UI and MP is central to the very notion of a logical rule, making UI and MP unadoptable.

So UI and MP presuppose themselves such that they are self-governing, and it is this feature that prevents them from being adoptable. These selfgoverning rules are, as Kripke puts it, 'completely useless' ${ }^{31}$ Nothing can be derived from the rules, nor can they be utilized, without a prior application of themselves. I have only outlined examples using UI and $\mathrm{MP},{ }^{32}$ but the issue that the AP picks up on is far more general. All logical rules require one to already know how and when to apply UI and MP, since all other logical rules presuppose them. If all other rules are derived (in some way) from them, then any problem with them will impact on the other rules. It thus seems all rules are not just derived from UI and MP, but rather they are governed by UI and MP. So, directly, UI and MP are unadoptable, and all other logical rules of inference become indirectly unadoptable as a result. This is because UI and MP are needed in order to infer in accordance with any logical rule of inference.

So, according to the AP, adopting a logic is impossible. ${ }^{33}$ But what exactly does adopting a logic mean? Padró defines adopting a logic in the following way:

Adoption of a logical rule $=$ acceptance of the rule + practice of inferring in accordance with that rule in virtue of accepting that rule. $^{34}$

\footnotetext{
${ }^{31}$ Kripke (1974a) as quoted in Padró (2015) p112.

${ }^{32}$ Padró (2015) fn.52 p37: "I will only talk about UI and MP. The former is Kripke's preferred example and the latter is Carroll's own example (and also the case that is most widely discussed in the literature), but of course it would be interesting to see which other principles are directly affected by this argument. That task, however, falls outside the scope of what I set out to do."

${ }^{33}$ It is not clear what the consequences of this are for the issue of the revisability of logic. There could potentially be ways to revise ones logic that doesn't require the utility of the rules in question, so that the reason for ones revision will not be in virtue of the rule itself.

${ }^{34}$ Padró (2015) p32. There may be other ways to adopt a logic that do not meet this definition and thus may not be problematic, e.g. undergoing classical conditioning to infer a certain way.
}

Australasian Journal of Logic (16:7) 2019, Article no. 3 
Given this characterization, adoption cannot be useless. The practice of inferring is a necessary part of the adoption process, and so if the acceptance of the rule does not lead to any practice of inferring in accordance with it then the rule cannot be said to have been adopted. Using Padró's definition of adoption, we can identify the three elements of the adoption process as being:

(1) The acceptance of an inference rule $R$

(2) The practice of inferring in accordance with that rule $R$

(3) Doing 2 in virtue of 1

In order to adopt a logic, one must successfully do all of 1,2 , and 3 , where each is necessary and together are jointly sufficient for capturing the adoption of a logical rule. Part 3 ensures that the practice of inferring is a rational rule-governed process, which begins in and is explained by the acceptance of a logical rule. 3 is therefore the element that gives the rule a certain use, since it is this usage that ensures that the practice of inferring happens because (or as a result) of the acceptance of the rule. Without 3 in the process, 1 and 2 amount to the practice merely conforming to the rule, and the rule plays no guiding role in shaping the practice. What 3 adds to 1 and 2 is the rule being the reason for the conformance, such that the rule is followed. It is this role of the rule that the AP shows to be problematic - the rule cannot be used as the reason for inferring in a certain way. The AP aims to show that logical rules cannot be utilized without the practice of inferring being previously established, and as such the rules of inference do not 'guide' our practice of inferring. And the reason why, I have argued, is due to the self-governance of UI and MP.

\section{The fall of exceptionalism: Kripke vs Carnap}

So far then, we have explored Quine's use of Carroll's puzzle against Carnap, and Kripke's use of it against Quine. This led us to Padró's interpretation of Kripke, in the form of the AP. Underlying all of these problems was the issue of having to use logic in order to deduce anything from logic, and I have demonstrated that this is due to self-governance. Kripke used this argument specifically against the anti-exceptionalist view of logic, however we will see that it is just as effective against the exceptionalist view of logic too. This is because Kripke claimed that Quine's objection against Carnap can be used 
against Quine himself, and in virtue of it being 'exactly the same', Kripke's objection should work against Carnap too. Therefore, the AP will apply to exceptionalism, as well as anti-exceptionalism, about logic. In this section of the paper I will show how Carnapian exceptionalism is susceptible to the AP.

Carnap thought that we could adopt one logic or another, or one language or another, or one linguistic framework or another, and that we have a choice between the options. ${ }^{35}$ We should be tolerant of the options, and we make a pragmatic choice between them. Carnap's principle of tolerance allows us to adopt any logic we choose, for as long as it is helpful for us to do so, without demanding that it is 'correct'. The principle of tolerance states that 'in logic, there are no morals', ${ }^{36}$ and so one is free to choose and adopt whichever they please as long as they can demonstrate the utility of that choice. So it seems that Carnap demands both the possibility to adopt different logics and for the logics to have utility. As we saw, the AP targets views that give logical rules such utility and allow for a choice regarding their adoption. As Kripke says:

The Carnapian tradition about logic maintained that one can adopt any kind of laws for the logical connectives that one pleases. This is a principle of tolerance, only some kind of scientific utility should make you prefer one to the other, but one is completely free to choose. Of course, a choice of a different logic is a choice of a different language form. Now, here we already have the notion of adopting a logic... As I said, I don't think you can adopt a logic. ${ }^{37}$

So, Kripke takes Carnap's notion of adopting a logic as being of the sort that he takes to be impossible, thus leaving Carnap's exceptionalist position just as much of a target of the AP as Quine's anti-exceptionalist position is.

However, in order for Carnap to truly be a target of the AP, he must hold exactly what the AP takes to be problematic, namely that rules have a guiding role in inference, such that the inference in accordance with the rule is in virtue of the acceptance of the rule. Does Carnap hold such a view? What is it within Carnap's position that makes it susceptible to the AP? Is Carnap really committed to the rules having a certain utility in inference? To be committed to the rules having such a utility, Carnap would need to be committed to a view where the adoption of logical rules is

\footnotetext{
${ }^{35}$ Carnap (1937)

${ }^{36}$ Ibid.

${ }^{37}$ Kripke (1974a) as quoted in Padró (2015) p113.
} 
possible, where adoption is defined as the acceptance of a logical rule and the practice of inferring in accordance with that rule in virtue of accepting that rule. Specifically then, Carnap will need to be committed to a position on rule-following. Even though Carnap does not make an explicit statement about rule-following, his view of logic may imply a problematic role for the rules as being utilized, which would make him susceptible to the AP. If the rules are not followed, then they are superfluous and have no pragmatic utility in inference, and since Carnap requires their utility to be demonstrated in order for them to be adopted, then Carnap is implicitly committed to a position whereby the rules are practical to accept in being followed. I take it that Carnap's point is that we adopt rules when we find them useful. We don't need to demonstrate usefulness in the sense of proving this, but rather the fact of their adoption is evidence that we found them to be useful. As such, Carnap is committed to the utility of logical rules.

Quine likewise says that the acceptance of the rule must have scientific fertility and the use of it must lead to successful predictions, and as such be pragmatic:

Carnap, Lewis, and others take a pragmatic stand on the question of choosing between language forms, scientific frameworks; but their pragmatism leaves off at the imagined boundary between the analytic and the synthetic. In repudiating such a boundary I espouse a more thorough pragmatism. Each man is given a scientific heritage plus a continuing barrage of sensory stimulation; and the considerations which guide him in warping his scientific heritage to fit his continuing sensory promptings are, where rational, pragmatic. ${ }^{38}$

The totality of our so-called knowledge or beliefs, from the most casual matters of geography and history to the profoundest laws of atomic physics or even of pure mathematics and logic, is a man-made fabric which impinges on experience only along the edges. ${ }^{39}$

Together with the point that this 'man-made fabric' is ultimately a tool for prediction, Quine also states that:

As an empiricist I continue to think of the conceptual scheme of

\footnotetext{
${ }^{38}$ Quine (1951) p43

${ }^{39}$ Ibid. p39
}

Australasian Journal of Logic (16:7) 2019, Article no. 3 
science as a tool, ultimately, for predicting future experience in the light of past experience. ${ }^{40}$

Presumably then, for both Carnap and Quine, we infer in accordance with the rules because we find it practical to do so. Does this amount to inferring in accordance with the rules in virtue of our acceptance of the rules? Perhaps. If we can say that our acceptance of the rules simply amounts to our judgment that it is practical to follow such rules, and it is in virtue of our judgement that it is practical to follow the rules that we then infer in accordance with those rules. Thus both Carnap and Quine are susceptible to the AP by requiring the rules to have a certain role in inference, where the rules have pragmatic utility.

\section{The return of Rule-Circularity}

Related to the AP is that, to follow any rule successfully requires two steps, first to know when the rule applies, and second to know how to apply it. The first step is to identify a structure and the appropriate rule for that structure, and the second step is to use that rule to infer a conclusion. As Wright describes:

Correctly applying a rule to a new case will, it is natural to think, typically involve a double success: it is necessary both to apprehend relevant features of the presented situation and to know what, in the light of those apprehended features, will fit or fail to fit the rule. ${ }^{41}$

In the AP this first step employs exactly the same rule as the second step. When we are required to employ the same rule in figuring out where and how it applies, when we have never applied it before (like Harry), we are left static. The required rule would be the same in the two steps because that rule is self-governing, since in order to understand that rule we make use of that rule.

This self-governing nature of logical rules such as UI and MP manifests not only in the AP, but also in the justification problem of Rule-Circularity, which too seems to cut across the exceptionalist/anti-exceptionalist divide (despite being most prominently posed for the exceptionalist). The problem of Rule-Circularity is roughly that the justification of any logical rule will

\footnotetext{
${ }^{40}$ Quine (1953) p42

${ }^{41}$ Wright (1989) p255
}

Australasian Journal of Logic (16:7) 2019, Article no. 3 
have to appeal to the basic logical rules, and so the justification of those basic logical rules will end up appealing to themselves. Let us for now consider UI and MP to be basic logical rules. Naturally, if these rules already govern themselves, then they will appeal to themselves for justification, just as they do in application. Both the AP and Rule-Circularity are thus manifestations of self-governance. Not only can we not justify UI and MP unless by means of themselves but we also cannot utilize them, and the reason why is due to their self-governance rather than a specific epistemological view about whether logic is exceptional or not.

The problems caused by the self-governance of logical rules will not cease by simply appealing to a different account of how the rules are justified. Therefore the targets of such problems cannot be selective to include only either the exceptionalist or anti-exceptionalist. Boghossian similarly argues that the issue of Rule-Circularity counts against any view of the warrant of logic, and cannot be used solely against the a priori exceptionalist as it has been previously:

What I do not see.... is how [Rule-Circularity] can be used to motivate an alternative epistemology for logic, one that is empirical in nature. For if we are barred from supposing that reasoning using a given logical principle can reconstruct an a priori warrant for that very principle, are we not equally barred from supposing that it could reconstruct an empirical warrant for that principle? Yet would not any empirical account of our warrant for believing the core principles of logic inevitably involve attributing to us reasoning using those very principles? ${ }^{42}$

Contrary to what some philosophers seem to think, then, the ban on circular justifications of logic cannot be used selectively, to knock out only a priori accounts of our warrant for logic. If it is allowed to stand, I do not see how it can be made to stop short of the very severe conclusion that we can have no warrant of any kind for our fundamental logical beliefs - whether of an a priori or a posteriori nature. ${ }^{43}$

It thus looks like the issues in rule-following, the AP, and Rule-Circularity all have the self-governance of certain logical rules in common. In light of this, more attention needs to be paid to their self-governance if we are to

\footnotetext{
${ }^{42}$ Boghossian (2000) p232-233.

${ }^{43}$ Ibid. p234.
} 
understand and resolve general problems in the epistemology of logic. So despite the AP and the problem of Rule-Circularity being posed specifically for anti-exceptionalist and exceptionalist positions respectively, the issues actually cut across that distinction given their common cause of self-governing rules.

\section{The morals of the story}

The AP applies to both Quine (as shown in section 3) and Carnap (as shown in section 5), who hold anti-exceptionalist and exceptionalist views of logic, respectively. Therefore, the AP is indifferent to the status or justification of logic - it simply does not matter whether the logical rules are empirical with no privileged status, or conventional with a privileged status, since the AP is concerned with how such rules get put into practice, seemingly regardless of their relative place in the system. It seems that one could not come to infer in accordance with a certain logical rule in virtue of accepting that rule, and it makes no difference whether the acceptance of the rule were empirical or not. I have shown that the AP is a problem for anyone who suggests that logical rules can be adopted whether on conventional (or more broadly exceptionalist) grounds or from a naturalist (or more broadly anti-exceptionalist) stance. The AP thus cuts across the exceptionalist/anti-exceptionalist boundary, and I argue that the source of the problem is in those rules being self-governing.

We saw that Harry cannot adopt UI, and we saw that the Tortoise refuses to adopt MP. Does it matter whether UI and MP are true by convention? No - this would just cause the problem outlined by Quine against Carnap. Does it matter whether UI and MP are empirically true? No - this would just cause the problem outlined by Kripke against Quine. So regardless of whether logic is exceptional or not, there will be the issue of putting that logic into practice via rational acceptance. The AP can thus be formulated against both exceptionalist and anti-exceptionalist positions, showing that the breadth of impact that the AP has is thus not limited to anti-exceptionalism as Kripke had articulated it. Therefore, the moral that we can draw from this diagnosis of the AP is that it does not discriminate among different interpretations of the truth of logical rules, but rather demonstrates a fundamental issue in the utility of logical rules, regardless of how those rules may be justified. The AP is indifferent towards how the rules are justified, but rather targets how those rules are used. Thus the AP does not support or attack positions because of their status of logic.

The target of Kripke's objection was Quinean anti-exceptionalist positions, 
as Kripke argued that it was the naturalist empiricist picture that caused the problem that we find in the AP. Against this, I have argued that the true cause of the problem was the self-governance of the logical rules, which is what prevented their adoption in either a naturalist empiricist picture or otherwise. However, given that Kripke claimed that his objection to the Quinean picture was the same as that which Quine posed against the Carnapian picture, it shows that Kripke may have thought there is something more fundamental doing the work in the AP rather than the naturalist empiricism. Padró makes a similar observation in order to demonstrate the breadth of the AP:

Thus, it seems that the Kripke-Carroll argument not only goes against views on the empiricist tradition. It also goes against views that favor the a priori and are usually considered part of the rationalist tradition. And it also goes against Kripke's own views on the role of intuitions. ${ }^{44}$

And elsewhere, Padró states:

We should emphasize that the problem here is general, it does not depend on the specific justification proposals we have discussed: the point is not that there will be inferential transitions that are not covered by the justification proposal on offer. It really doesn't matter what kind of justification we favor, whether we claim that logical principles are knowable a priori or a posteriori, or whether we claim that our knowledge of them is unjustifiable. Even their truth or validity is beside the point. The issue is what role these rules can have in actual instances of inferring. ${ }^{45}$

According to Padró's interpretation then, at the core of Quine's and Kripke's usage of Carroll's puzzle was the more familiar problem of rule-following and the nature of inference, which is a problem that we all must face, and not just those who hold a Quinean anti-exceptionalist view of logic.

What I contribute to the discussion is explicitly connecting the AP directly to the exceptionalist/anti-exceptionalist debate, showing that the AP applies to both positions, and furthermore showing why it applies to both positions. Instead of attributing the core issue to be with regard to rule-following and the nature of inference, I attribute it to the nature of the logical rules themselves. Given that logical rules are universal and conditional in their structure, the

\footnotetext{
${ }^{44}$ Padró (2015) p156

${ }^{45}$ Ibid. p195
}

Australasian Journal of Logic (16:7) 2019, Article no. 3 
rules that govern universal and conditional structures will be self-governing. I have argued that this self-governance is what is at the heart of the AP, which is what causes the unadoptability of certain logical rules despite the system of logic at hand or its justification. The moral of the story therefore is that the AP is devastating, not just to the anti-exceptionalist, but to the exceptionalist too, due to the more fundamental issue of the self-governance of basic logical rules.

\section{Conclusion}

Carroll's puzzle can be interpreted in many ways. Quine uses it and directs it against the status of logic being conventional, whereas Kripke uses it and directs it against the status of logic being unprivileged. Quine showed that Carnap could not deduce anything from the logical rules if they were conventional, whilst Kripke showed that Quine could not deduce anything from the logical rules if they were empirical. The similarity between these uses of Carroll's puzzle is thus not the target, but rather the problem of using the logical rules to deduce things from them. I have argued that this problem of utility manifests as a result of the basic logical rules of inference being self-governing such that nothing can be deduced from them without a prior usage of themselves. And since on both the Quinean anti-exceptionalist and Carnapian exceptionalist models of logic the rules are meant to have a certain practical utility, both models are thus susceptible to the AP. The AP therefore does not discriminate between the status or justification of logic, given that it proves problematic regardless of whether logic is seen as being privileged, unprivileged, analytic, or empirical. In relation to the roots of the AP, Kripke stated in disbelief that "somehow people haven't realized how deep this kind of issue cuts," 46 yet now it cuts even deeper, proving destructive for both anti-exceptionalist and exceptionalist positions. And what I have attempted to show in this paper is the otherwise unarticulated reason why it cuts so deep: For as long as we utilize self-governing rules like UI and MP, there will be trouble. ${ }^{47}$

\footnotetext{
${ }^{46}$ Kripke (1974a) as quoted in Padró (2015) p113.

${ }^{47}$ Special thanks goes to Romina Padró, Michael Devitt, Mary Leng, and Tom Stoneham, for their very helpful comments on earlier drafts of this paper, and to Saul Kripke for his support of my writing this paper. Also many thanks to the audiences of the Logic and Metaphysics workshop at the City University of New York Graduate Center, the Dublin Philosophy Research Network Philosophy of Language workshop at Trinity College Dublin, the Logic as Science conference at the University of Bergen, the Research Day at the University of Southampton, and the Mind and Reason group at the University of York,
} 


\section{References}

Boghossian, P. (2000). Knowledge of logic. In Boghossian, P. and Peacocke, C., editors, New Essays on the A Priori, pages 229-254. OUP, Oxford.

Carnap, R. (1937). The logical syntax of language. Routledge \& Kegan Paul Ltd, New York.

Carroll, L. (1895). What the Tortoise said to Achilles. Mind, 4:278-280.

Ebbs, G. (2011). Carnap and quine on truth by convention. Mind, 120(478):193-237.

Finn, S. (2019). Limiting logical pluralism. Synthese.

Hjortland, O. T. (2017). Anti-exceptionalism about logic. Philosophical Studies, 174(3):631658.

Kripke, S. (1974a). Princeton lectures on the nature of logic. Transcription.

Kripke, S. (1974b). The question of logic. Transcription of a lecture given at the University of Pittsburg.

Padró, R. (2015). What the Tortoise Said to Kripke: the Adoption Problem and the Epistemology of Logic. PhD thesis, CUNY.

Putnam, H. (1969). Is logic empirical? In Cohen, R. S. and Wartofsky, M. W., editors, Boston Studies in the Philosophy of Science: Proceedings of the Boston Colloquium for the Philosophy of Science 1966/1968, pages 216-241. Springer, Dordrecht.

Quine, W. V. (1936). Truth by convention. In Rosser, B., editor, Philosophical essays for Alfred North Whitehead, pages 90-124. Longmans, Green and Co., New York.

Quine, W. V. (1951). Two dogmas of empiricism. Philosophical Review, 60(1):20-43.

Quine, W. V. (1953). From a logical point of view. Harvard University Press, Cambridge, MA.

Wright, C. (1989). Wittgenstein's rule-following considerations and the central project of theoretical linguistic. In George, A., editor, Reflections on Chomsky. Blackwell, New York.

Yablo, S. (1998). Does ontology rest on a mistake? Aristotelian Society Supplementary Volume, 72(1):229-262.

where I presented ideas that derive from this paper. This paper was completed during a project that has received funding from the European Research Council (ERC) under the European Union's Horizon 2020 research and innovation programme, under grant agreement number 679586.

Australasian Journal of Logic (16:7) 2019, Article no. 3 